\title{
Robust Output Tracking Control of a Surface Vessel
}

\author{
DongBin Lee, Enver Tatlicioglu, Timothy C. Burg*, Darren M. Dawson
}

\begin{abstract}
In this paper, tracking control of a three degreeof-freedom marine vessel is examined. The primary motivation for this work is the compensation needed for the added mass common to surface vessels, resulting in an asymmetric inertia matrix. Two control schemes are considered: a fullstate feedback controller and an output feedback controller. Numerical simulation results are shown to demonstrate the validity of these proposed controllers.
\end{abstract}

\section{Introduction}

Research into the control of marine surface vessels could be loosely categorized as maneuvering [1,2], dynamic positioning [3,4], tracking (including path following or way-point tracking) $[6,8]$, and more recently formation control [5]. From a control perspective, the properties of the dynamic model of the surface vessel are of great importance. Specifically, the symmetry and the positive definiteness of the inertia matrix are an important assumption often used in control development. The inertia matrix of a vessel is commonly defined to be equal to the sum of the rigid-body inertia matrix and the added mass. The rigid-body inertia matrix is strictly a symmetric matrix. The added mass terms result from hydrodynamic forces and moments due to motion of the vessel body and from the interaction with the fluids and waves. In surface vessel control, the added mass matrix can easily become asymmetric, especially for higher relative velocity. This asymmetry in the added mass terms will result in an asymmetric inertia matrix in the ship model, which may cause system instability or a failure in meeting the control objectives if ignored.

As the foundation of this work, previous closely related work is described. In [2], Skjetne et al. considered maneuvering control of three degree-of-freedom (3 DOF) marine vessel and presented experiment results for the Cybership II. In [6], Do and Pan presented a global tracking controller of an underactuated vessel where the system matrices are positive definite but nonzero off-diagonal terms and Do in [7] proposed robust and adaptive output feedback controllers for positioning of a surface vessel assumed the system matrices to be positive definite at low speed. In [8], Behal et al. utilized a

This work has been supported in part by a DOE Contract, a Honda Corporation Grant.

D. Lee, T. C. Burg, D. M. Dawson are with the Dep't of Electrical and Computer Engineering, Clemson University, Clemson, SC 29634, USA. email: dlee@clemson.edu, tburg@clemson.edu (*corresponding author), darren.dawson@ces.clemson.edu. E. Tatlicioglu was Dep't of Electrical \& Computer Engineering, Clemson University. He is now with the Dep't of Electrical and Electronics Engineering, Izmir Institute of Technology, Urla, Izmir 35430, Turkey. email: enver@envertatlicioglu.com high-gain observer in the design to control underactuated surface vessels with nonintegrable dynamic models where the inertia matrix was diagonal. However, a few researchers have addressed the asymmetry of the inertia matrix. For example, Skjetne et al. in [2] developed the Coriolis-centripetal and damping model of the ship for the asymmetric added mass matrix, but the mass-related parameters were used in the symmetric form at the experiment, which may be violated at higher operating speeds.

In this paper, we focus on tracking control of a $3 \mathrm{DOF}$ surface vessel. The dynamic model of the ship is assumed to be uncertain and the added mass terms are considered to be asymmetric which results in an asymmetric inertia matrix. To address this problem, the dynamic model of the ship is modified to have a symmetric and positivedefinite inertia matrix. The novelty of this modification is the multiplication of the system dynamic model in [2] with an upper triangular matrix which results in a model with a symmetric inertia matrix. After this modification, the resulting dynamic model becomes a special case of the multi-input multi-output system that was considered in [10] (see equation 12 in $[10]^{1}$ ). Next, the robust full-state feedback (FSFB) and output feedback (OFB) control strategies in [10], which were designed for general class of multi-input multi-output nonlinear systems, are tailored to fit this dynamic surface vessel model.

The paper is organized as follows: Section II presents a dynamic and a kinematic model of the 3 DOF surface vessel. The error system development and the control strategies are provided in Section III. The numerical simulation results are shown in Section IV followed by conclusions in Section V.

\section{System Model}

In this section, the system model and relevant properties are discussed. The dynamic and kinematic models of a 3 DOF surface vessel expressed in the body-fixed frame, $B$, are given as $[1,2]$

$$
\begin{aligned}
M \dot{\nu}+C \nu+D \nu & =\tau \\
\dot{x} & =R \nu
\end{aligned}
$$

where the vector $\dot{x}(t) \in \mathbb{R}^{3}$ represents the position and orientation rate in which $x=\left[x_{p}, y_{p}, \psi\right]^{\top}$ denotes the

\footnotetext{
${ }^{1}$ The robust control development in [9] is similar to [10] with a minor modification in the matrix decomposition (see Lemma 1 in both [9] and [10]). In that sense, throughout this paper we will refer to [9], however, the reader is also referred to [10].
} 
linear position $\left(x_{p}, y_{p}\right)$ along the $X$ - and the $Y$-axes and the yaw angle $(\psi)$. The vectors $v_{r}(t), \nu(t)=[u, v$, $\dot{\psi}]^{\top}$, and $\dot{\nu}(t) \in \mathbb{R}^{3}$ denote the relative velocity between the fluids and vessel and the velocity and acceleration of the rigid-body ship, respectively. $M(\psi), C\left(\nu, \nu_{r}\right)$, $D\left(\nu, \nu_{r}\right) \in \mathbb{R}^{3 \times 3}$ represent the inertia matrix, centripetal and Coriolis force, and hydrodynamic damping terms, respectively. In the subsequent control development, $M(\cdot), C(\cdot)$, and $D(\cdot)$ are assumed to be uncertain and continuously differentiable up to their second time derivatives. In (1), $\tau(t) \in \mathbb{R}^{3}$ represents the control input vector which has the following form

$$
\tau=\left[\begin{array}{lll}
\tau_{1}, & \tau_{2}, \tau_{3}
\end{array}\right]^{\top}
$$

where $\tau_{1}(t)$ and $\tau_{2}(t) \in \mathbb{R}^{1}$ are the translational forces in the $X$ - and $Y$-directions, respectively and $\tau_{3}(t) \in \mathbb{R}^{1}$ is the moment about the $Z$-axis. The matrix, $R(\psi) \in$ $S O(3)$, denotes the rotation matrix, containing the yaw angle, about the $Z$-axis. The coordinate frame of the surface vessel is presented in Figure 1 , where $B$ is the body-fixed reference frame of the vessel and a fixed inertial frame, approximated by the earth-fixed frame (North-East-Down convention), is denoted by $I$. The

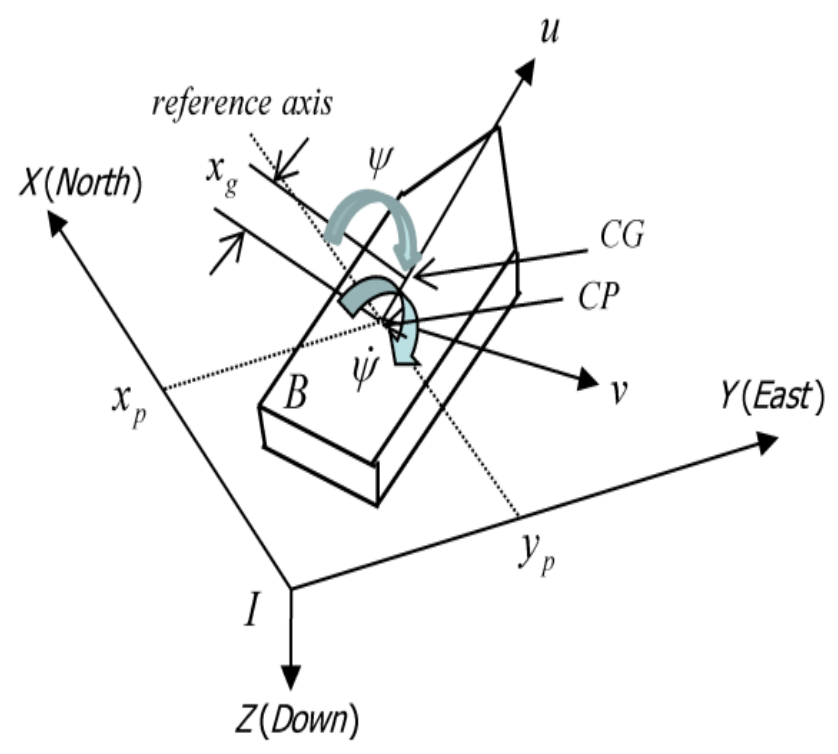

Fig. 1. Diagram of a Surface Vessel

states are measured from the center point (CP) of the ship frame expressed in $B$ and $x_{g}$ denotes the distance between the center point and the center of gravity (CG) of the ship.

The inertia matrix, $M(\cdot)$, of the ship is defined as [2]

$$
M \triangleq M_{R B}+M_{A}
$$

where $M_{R B}(\cdot) \in \mathbb{R}^{3 \times 3}$ represents the rigid-body symmetric inertia and $M_{A}(\cdot) \in \mathbb{R}^{3 \times 3}$ accounts for the asymmetric added mass. Since $M_{A}(\cdot)$ is asymmetric the inertia matrix of the surface vessel, $M(\cdot)$, is also an asymmetric matrix.
To facilitate the subsequent control development, the dynamic model in (1) will now be modified to obtain a symmetric inertia matrix. There will exist an upper diagonal matrix, $T(\cdot) \in \mathbb{R}^{3 \times 3}$, such that the multiplication of $T(\cdot)$ and $M(\cdot)$ results in a symmetric, positive definite matrix, denoted by $M_{s}(\cdot) \in \mathbb{R}^{3 \times 3}$. After multiplying (1) with $T(\cdot)$, the following expression is obtained

$$
M_{s} \dot{\nu}=-T(C+D) \nu+T \tau \text {. }
$$

To further modify the dynamic model, the time derivative of (2) is obtained in the following form

$$
\dot{\nu}=R^{\top} \ddot{x}-R^{\top} \dot{R} R^{\top} \dot{x}
$$

where the property of the rotation matrix that $R^{-1}(\psi)=$ $R^{\top}(\psi)$ was utilized. Substituting (6) into (5) yields

$$
M_{s} R^{\top} \ddot{x}=\left[M_{s} S_{3}(\dot{\psi})-T(C+D)\right] R^{\top} \dot{x}+T \tau
$$

where the time derivative of the orientation matrix, denoted by $\dot{R}(\psi)$, and a skew-symmetric matrix $S_{3}(\dot{\psi}) \in$ $\mathbb{R}^{3 \times 3}$ can be calculated as follows

$$
\dot{R}(\psi)=R S(\dot{\psi}), S_{3}(\dot{\psi})=\left[\begin{array}{ccc}
0 & -\dot{\psi} & 0 \\
\dot{\psi} & 0 & 0 \\
0 & 0 & 0
\end{array}\right]
$$

After premultiplying (7) with $R(\psi)$, the following model is obtained

$$
\bar{M}(\psi) \ddot{x}=\bar{C}\left(x, \dot{x}, \nu, \nu_{r}\right) \dot{x}+R T \tau
$$

where $\bar{M}(\psi)$ and $\bar{C}\left(x, \dot{x}, \nu, \nu_{r}\right) \in \mathbb{R}^{3 \times 3}$ are defined as

$$
\begin{gathered}
\bar{M} \triangleq R M_{s} R^{\top} \\
\bar{C}=R\left[M_{s} S_{3}(\dot{\psi})-T(C+D)\right] R^{\top} .
\end{gathered}
$$

It should be noted that the form of (9) was motivated by the dynamic model in [9], [10] and hence, solving the same control problem.

Propertyhe:matrix $\bar{M}(\psi)$ is positive definite, symmetric, and satisfies the following inequalities

$$
\lambda_{1}\|\xi\|^{2} \leq \xi^{T} \bar{M} \xi \leq \lambda_{2}\|\xi\|^{2}, \forall \xi \in \mathbb{R}^{3}
$$

where $\lambda_{1}, \lambda_{2} \in \mathbb{R}^{1}$ are positive bounding constants.

\section{Control Development}

\section{A. Full-State Feedback Control}

The subsequent development is based on the assumption that all the states of the vessel are measurable. 
1) Error System Development

: The tracking error for position and orientation, denoted by $e_{1}(t) \in \mathbb{R}^{3}$, is defined as

$$
e_{1} \triangleq x_{d}-x
$$

where $x_{d}(t) \in \mathbb{R}^{3}$ is the desired trajectory. For the subsequent stability analysis, the desired trajectory and its first and second time derivatives are assumed to be bounded (i.e., $x_{d}(t), \dot{x}_{d}(t)$, and $\ddot{x}_{d}(t) \in \mathcal{L}_{\infty}$ ). To facilitate the subsequent error system development, a filtered error, denoted by $e_{2}(t) \in \mathbb{R}^{3}$, is defined as

$$
e_{2} \triangleq \dot{e}_{1}+e_{1} \text {. }
$$

In order to simplify the error system and to facilitate the stability analysis, a filtered tracking error is introduced as

$$
r \triangleq e_{1}+e_{2} .
$$

The dynamics of $r(t)$ can be obtained as follows

$$
\dot{r}=\ddot{x}_{d}-\ddot{x}+2 \dot{e}_{1}
$$

where the second time derivatives of (13) and (14) were utilized. After premultiplying (16) with $\bar{M}(\psi)$, we can obtain the following expression

$$
\bar{M} \dot{r}=\bar{M} \ddot{x}_{d}-\bar{C} \dot{x}-R T \tau+2 \bar{M} \dot{e}_{1}
$$

where (9) was utilized. The expression in (17) can be rearranged after adding and subtracting the terms $\frac{1}{2} \dot{\bar{M}} r$ $(t), e_{2}(t)$, and $R \tau(t)$ to the right-hand side as

$$
\bar{M} \dot{r}=N-R \tau-\frac{1}{2} \dot{\bar{M}} r-e_{2}-R\left(T-I_{3}\right) \tau
$$

where the auxiliary signal $N(\cdot) \in \mathbb{R}^{3}$ is defined by

$$
N \triangleq \bar{M} \ddot{x}_{d}-\bar{C} \dot{x}+2 \bar{M} \dot{e}_{1}+\frac{1}{2} \dot{\bar{M}} r+e_{2} .
$$

To facilitate the control development, the open-loop error dynamics can be obtained from (18) as

$$
\bar{M} \dot{r}=\tilde{N}+N_{d}-\frac{1}{2} \dot{\bar{M}} r-e_{2}-\left[\begin{array}{c}
\Lambda \\
0
\end{array}\right]-\left[\begin{array}{c}
\Phi_{d} \\
0
\end{array}\right]-R \tau
$$

where the signals $\Lambda(t), \Phi_{d}(t) \in \mathbb{R}^{2}$ are defined as

$$
\left[\begin{array}{c}
\Lambda+\Phi_{d} \\
0
\end{array}\right] \triangleq R\left(T-I_{3}\right) \tau
$$

where the third component of $\left(T-I_{3}\right)$ is zero (an example of $T(\cdot)$ is shown in the simulation section) and $N_{d}(\cdot)$, $\tilde{N}(\cdot) \in \mathbb{R}^{3}$ are defined as follows

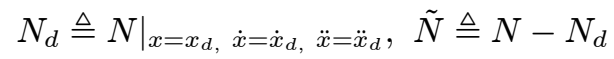

where the desired trajectory and its first two time derivatives are assumed to be bounded and hence $N_{d}(\cdot)$ and $\dot{N}_{d}(\cdot)$ are bounded signals.

Remark 1: The term $\tilde{N}(\cdot)$ in (22) is upper bounded as

$$
\|\tilde{N}\| \leq \rho_{N}(\|z\|)\|z\|
$$

where $\rho_{N}(\cdot)$ is a globally invertible, non-decreasing function and the auxiliary error vector $z(t) \in \mathbb{R}^{9}$ is defined as

$$
z(t)=\left[e_{1}^{\top}, e_{2}^{\top}, r^{\top}\right]^{\top} .
$$

2) Control Input

: Based on the open-loop error dynamics in (20) and the result in [9], the control input $\tau(t)$ is designed as

$$
\tau \triangleq R^{\top}\left(K+I_{3}\right) r+R^{\top} \hat{f}
$$

where $K \in \mathbb{R}^{3 \times 3}$ is a constant positive definite diagonal gain matrix and $\hat{f}(t) \in \mathbb{R}^{3}$ is a feedforward component that is introduced to compensate for $N_{d}(t)$ and $\Phi_{d}(t)$. After substituting (24) into (20), the following closedloop error system can be obtained

$$
\bar{M} \dot{r}=\Pi+\Psi_{d}-\frac{1}{2} \dot{\bar{M}} r-e_{2}-\left(K+I_{3}\right) r
$$

where $\Pi(t)$ and $\Psi_{d}(t) \in \mathbb{R}^{3}$ are auxiliary functions defined as follows

$$
\Pi \triangleq \tilde{N}-\left[\begin{array}{c}
\Lambda \\
0
\end{array}\right], \Psi_{d} \triangleq N_{d}-\left[\begin{array}{c}
\Phi_{d} \\
0
\end{array}\right]-\hat{f} .
$$

Note that since $\hat{f}(\cdot)$ is used to compensate for functions of the desired trajectory it is known that $\hat{f}(\cdot) \in \mathcal{L}_{\infty}$ apriori.

Remark 2: The controller proposed in (24) is an application of the previous theoretical development in [9] where the proposed control yields a semi-global, uniformly, and ultimately bounded (sGUUB) tracking result. Thus, the reader is referred to [9] for a detailed stability analysis.

Remark 3: The term $\hat{f}(\cdot)$ in (24) and (35) is not directly specified here but in practice it can be implemented in other ways including a neural network.

\section{B. Output Feedback Control}

The following development is based on the assumption that the position and the orientation of the ship, $x(t)$, is the only state that is measurable.

1) Observer Design

: To facilitate the subsequent control development, the auxiliary error vector, denoted by $z(t) \in \mathbb{R}^{6}$, is redefined as

$$
z(t)=\left[e_{1}^{\top}, r^{\top}\right]^{\top}
$$

where $e_{1}(t)$ and $r(t)$ are error signals defined in (13) and (15), respectively. The following expression can be obtained for the dynamics of $z(t)$

$$
\dot{z}=\left[\left(r-2 e_{1}\right)^{\top}, \dot{r}^{\top}\right]^{\top}
$$

where (13) and (14) were utilized.

An estimate of the unmeasurable, $z(t)$ in (27), is introduced as follows

$$
\hat{z} \triangleq\left[\hat{e}_{1}^{\top}, \hat{r}^{\top}\right]^{\top}
$$

where $\hat{e}_{1}(t), \hat{r}(t) \in \mathbb{R}^{3}$ are high-gain observers that are introduced to estimate the error signals $e_{1}(t)$ and $r(t)$, respectively [11]. The time derivative of (29) can be obtained as

$$
\dot{\hat{z}} \triangleq\left[\begin{array}{c}
\dot{\hat{e}_{1}} \\
\dot{\hat{r}}
\end{array}\right]=\left[\begin{array}{c}
\hat{r}-2 \hat{e}_{1}+\frac{\alpha_{1}}{\varepsilon}\left(e_{1}-\hat{e}_{1}\right) \\
\frac{\alpha_{2}}{\varepsilon^{2}}\left(e_{1}-\hat{e}_{1}\right)
\end{array}\right] .
$$


To facilitate the subsequent analysis, the following observer errors, denoted by $\eta_{1}(t)$ and $\eta_{2}(t) \in \mathbb{R}^{3}$, are introduced

$$
\eta_{1}=\frac{1}{\varepsilon}\left(e_{1}-\hat{e}_{1}\right), \eta_{2}=r-\hat{r} .
$$

The dynamics for the observer errors can be obtained as follows

$$
\dot{\eta}_{1}=\frac{1}{\varepsilon}\left(-\alpha_{1} \eta_{1}+\eta_{2}-2 \varepsilon \eta_{1}\right), \dot{\eta}_{2}=-\frac{\alpha_{2}}{\varepsilon} \eta_{1}+\dot{r}
$$

where (30) and (31) were utilized. After combining (32), the following simplified expression can be obtained

$$
\varepsilon \dot{\bar{\eta}}=A_{o} \bar{\eta}+\varepsilon g
$$

where $\bar{\eta}(t) \triangleq\left[\eta_{1}^{\top}, \eta_{2}^{\top}\right]^{\top} \in \mathbb{R}^{6}$ and the signals $g(t) \in \mathbb{R}^{6}$ and $A_{o} \in \mathbb{R}^{6 \times 6}$ are defined as follows

$$
A_{o}=\left[\begin{array}{cc}
-\alpha_{1} I_{3} & I_{3} \\
-\alpha_{2} I_{3} & O_{3}
\end{array}\right], g=\left[\begin{array}{c}
-2 \eta_{1} \\
\dot{r}
\end{array}\right] .
$$

2) Control Input

: Similar to (24) and the result in [9], the output feedback controller for $\dot{r}(t)$ in (34) is designed as follows

$$
\tau \triangleq R^{\top} \operatorname{sat}\left\{\left(K+I_{3}\right) \hat{r}\right\}+R^{\top} \hat{f}
$$

where $\operatorname{sat}\{\cdot\} \in \mathbb{R}^{3}$ represents the vector saturation function and $\hat{f}(t) \in \mathbb{R}^{3}$ is the feedforward term (see Remark 3). Substituting this control input into (20) yields the following closed-loop error system

$$
\bar{M} \dot{r}=\Pi+\Psi_{d}-\frac{1}{2} \dot{\bar{M}} r-e_{2}-\operatorname{sat}\left\{\left(K+I_{3}\right) \hat{r}\right\}
$$

where $\Pi(\cdot)$ and $\Psi_{d}(\cdot)$ were introduced in $(26)$.

Remark 4: Since the output feedback controller designed in (35) is a special case of the development in [9], a semi-global, uniformly, and ultimately bounded (sGUUB) tracking result can be inferred.

\section{Numerical Simulation Results}

Two numerical simulations were performed to show the validity of the proposed controllers. The rigid-body inertia matrix including the added mass terms are of the following form [2]

$$
M=\left[\begin{array}{ccc}
m+X_{\dot{u}} & 0 & 0 \\
0 & n_{a} & n_{d} \\
0 & n_{c} & n_{b}
\end{array}\right]
$$

where $n_{a}, n_{b}, n_{c}$, and $n_{d}$ are auxiliary terms that are defined as follows

$$
\begin{aligned}
& n_{a}=m-Y_{\dot{v}}, n_{b}=I_{z}-N_{\dot{r}} \\
& n_{c}=m x_{g}-N_{\dot{v}}, n_{d}=m x_{g}-Y_{\dot{r}} .
\end{aligned}
$$

From (37), it can be seen that the added mass matrix, denoted by $M_{A}(\cdot)$, has nonzero off-diagonal hydrodynamic damping terms (usually refered to as hydrodynamic derivatives). In [2], $Y_{\dot{r}}$ and $N_{\dot{v}}$ were set equal to zero which resulted in a symmetric inertia matrix. It is clear that if the values of $Y_{\dot{r}}$ and $N_{\dot{v}}$ are different, then the resulting matrix is asymmetric. For the simulation, the following values were chosen for these parameters

$$
Y_{\dot{r}}=0.0, N_{\dot{v}}=-1.0,
$$

which produce an asymmetric inertia matrix. Based on the inertia matrix in $(37)$, the following $T(\cdot)$ matrix is defined to modify the system dynamic model

$$
T=\left[\begin{array}{ccc}
1 & 0 & 0 \\
0 & \frac{-n_{a} n_{b}}{d}+\frac{n_{c}^{2}}{d} & \frac{n_{a} n_{d}}{d}+\frac{-n_{a} n_{c}}{d} \\
0 & 0 & \frac{n_{c} n_{d}}{d}+\frac{-n_{a} n_{b}}{d}
\end{array}\right]
$$

where $d$ is a non-zero auxiliary term defined as

$$
\begin{aligned}
d= & -m I_{z}+m N_{\dot{r}}+Y_{\dot{v}} I_{z}-Y_{\dot{v}} N_{\dot{r}}+\left(m x_{g}\right)^{2} \\
& -m x_{g} N_{\dot{v}}-m x_{g} Y_{\dot{r}}+Y_{\dot{r}} N_{\dot{v}} .
\end{aligned}
$$

The Coriolis-centripetal term $C(\cdot)$ in (1) is defined by combining the rigid-body matrix $C_{R B}(\nu) \in \mathbb{R}^{3 \times 3}$ and corresponding added mass $C_{A}\left(\nu, \nu_{r}\right) \in \mathbb{R}^{3 \times 3}$ as

$$
C\left(\nu, \nu_{r}\right)=\left[\begin{array}{ccc}
0 & 0 & c_{2} \\
0 & 0 & -c_{1} \\
-c_{2} & c_{1} & 0
\end{array}\right]
$$

where $c_{1}\left(\nu_{r}\right)=m u+\left(-X_{\dot{u}} u_{r}\right)$ and $c_{2}\left(\nu, \nu_{r}\right)=-m\left(x_{g} \dot{\psi}+\right.$ $v)+\left(Y_{\dot{v}} v_{r}+.5\left(Y_{\dot{r}}+N_{\dot{v}}\right) \dot{\psi}\right)$. The damping matrix $D(\cdot)$ in (1) is defined by combining the linear matrix term $D_{L}(\nu) \in \mathbb{R}^{3 \times 3}$ and nonlinear matrix $D_{N L}\left(\nu, \nu_{r}\right) \in \mathbb{R}^{3 \times 3}$ as

$$
D\left(\nu, \nu_{r}\right)=\left[\begin{array}{ccc}
d_{11} & 0 & 0 \\
0 & d_{22} & d_{23} \\
0 & d_{32} & d_{33}
\end{array}\right]
$$

where $d_{11}\left(\nu_{r}\right)=-X_{u}+\left(-X_{|u| u}\left|u_{r}\right|-X_{u u u} u_{r}^{2}\right)$, $d_{22}\left(\nu, \nu_{r}\right)=-Y_{v}+\left(-Y_{|v| v}\left|u_{r}\right|-Y_{r v}|\dot{\psi}|\right), d_{33}\left(\nu, \nu_{r}\right)=$ $-N_{r}+\left(-Y_{|v| v}\left|u_{r}\right|-Y_{|r| v}|\dot{\psi}|\right), d_{23}\left(\nu, \nu_{r}\right)=-Y_{r}+$ $\left(-Y_{|v| r}\left|u_{r}\right|-Y_{|r| v}|\dot{\psi}|\right)$, and $d_{32}\left(\nu, \nu_{r}\right)=-N_{v}+$ $\left(-N_{|v| v}\left|v_{r}\right|-N_{r v}|\dot{\psi}|\right)$. The saturator which limts the signals to upper and lower was used by \pm 100 for the control input both FSFB and OFB controllers. The other system parameters were obtained from the experimental results in [2]. The desired position and yaw trajectory are specified as below

$x_{d}(t)=\left[\begin{array}{c}10 \sin (0.1 t) \\ 10 \cos (0.1 t)\end{array}\right](m), \quad \psi_{d}(t)=-0.1 t(\mathrm{rad})$,

and the vessel was considered to be initially at rest in the following configuration $x(0)=\left[0.1,1,-\frac{\pi}{8}\right]^{\top}$. The surface vessel was specified to have the relative velocity, $v_{r}=[3,0,0]^{\top}$ where the relative velocity is assumed to have the constant surge speed, $3[\mathrm{~m} / \mathrm{s}]$, about the $\mathrm{X}$-axis.

\section{A. Full-State Feedback (FSFB) Control}

For the FSFB controller simulation, the constant control parameter was chosen as $K=$ $\operatorname{diag}\left\{\begin{array}{lll}500 & 500 & 100\end{array}\right\}$. In Figure 2, tracking the desired trajectory of the surface vessel in $X Y$-plane is demonstrated. In Figure 3, the position and yaw angle 


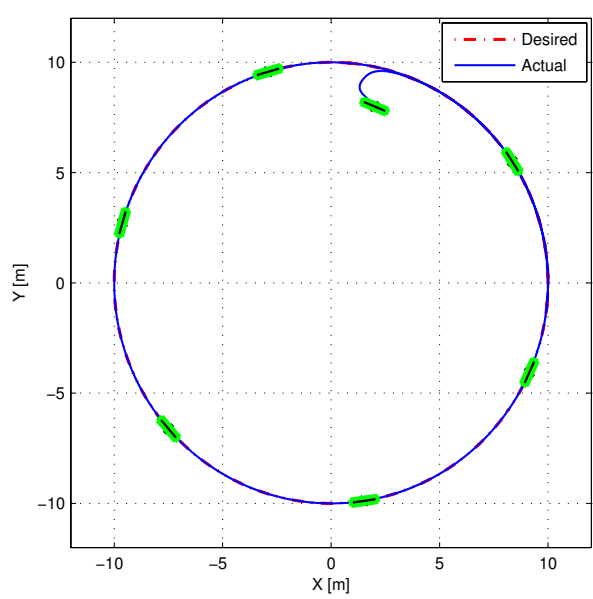

Fig. 2. Tracking demonstration in the XY-Plane (FSFB)

of the ship are presented along with their corresponding desired trajectories. The error signal $e_{1}(t)$ is presented in Figure 4. From Figure 4, it is clear that the surface vessel tracked the desired trajectory. The control input $\tau(t)$ is shown in Figure 5 .
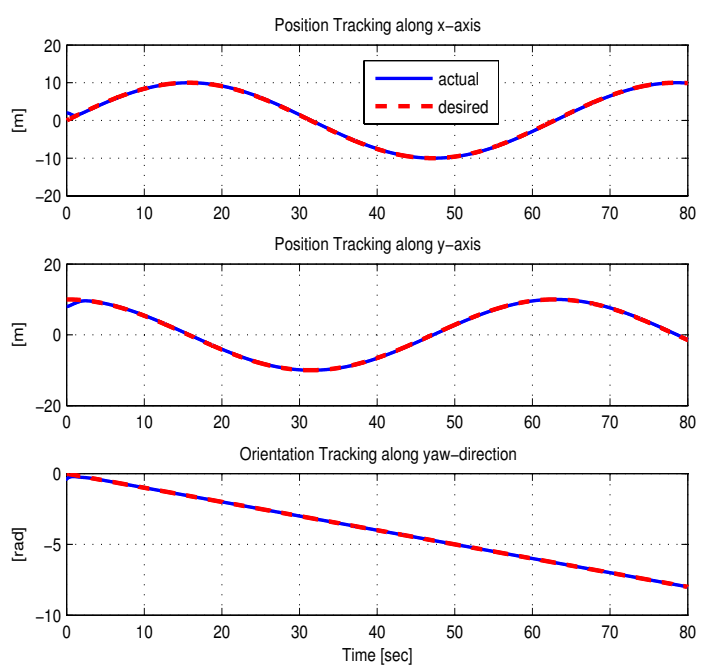

Fig. 3. Tracking demonstration (FSFB)

\section{B. Output Feedback (OFB) Control}

For the OFB controller, the constant control parameter was chosen as $K=\operatorname{diag}\left\{\begin{array}{lll}400 & 400 & 100\end{array}\right\}$ and the following control parameters were used $\alpha_{1}=100, \quad \alpha_{2}=100, \quad$ and $\varepsilon=0.1$. In Figure 6 , the position and the desired trajectory of the surface vessel in $X Y$-plane is demonstrated. Figure 7 shows the position and yaw angle of the ship along with their corresponding desired trajectories. Figure 8 displays the small, bounded tracking error signals. In Figure 9, the control input $\tau(t)$ is presented.
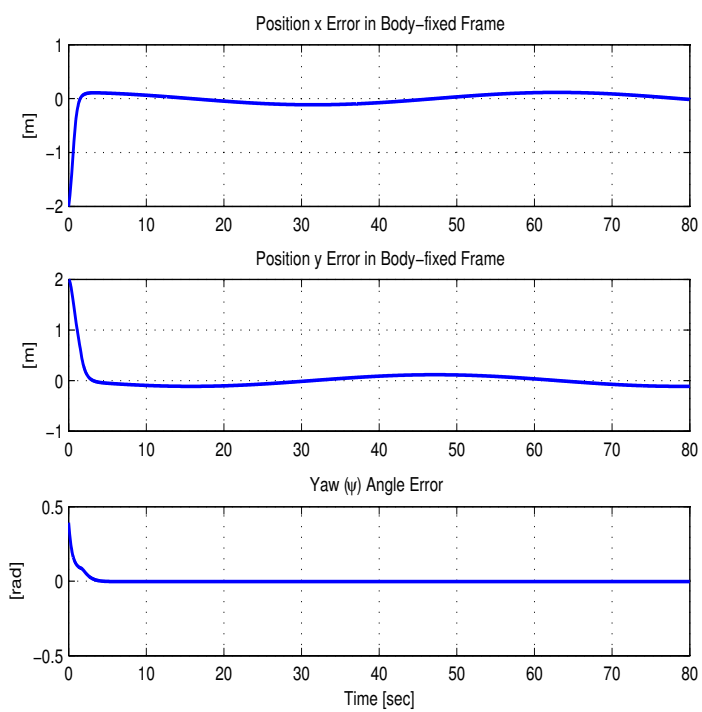

Fig. 4. Tracking Errors in Position $\left(x_{p}, y_{p}\right)$ and Yaw Angle $(\psi)$ (FSFB)
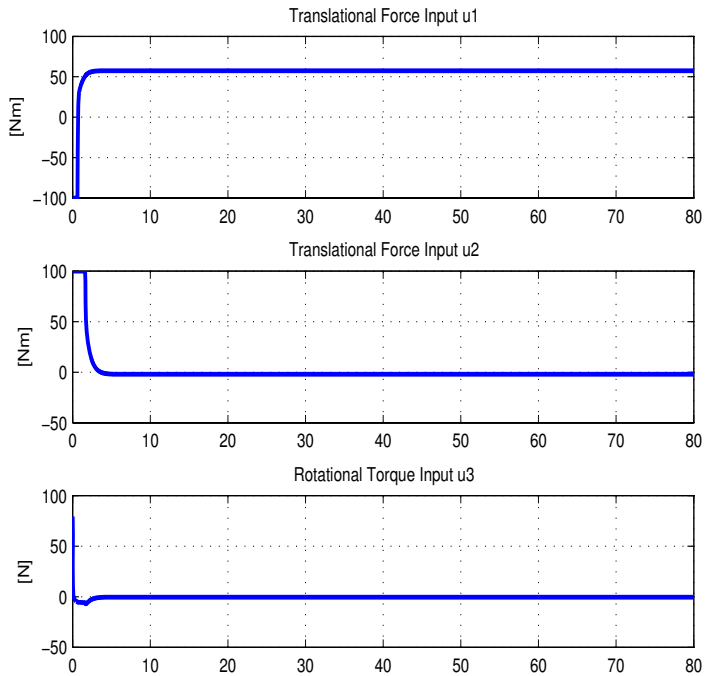

Fig. 5. Forces and Torque Input (FSFB)

\section{Conclusion}

The control problem of surface vessels with asymmetric inertia matrices was addressed. The significance of this work was the modification of the inertia matrix by pre-multiplying with an upper triangular matrix to obtain a symmetric form. Then, a full-state feedback and an output feedback controller were designed. Numerical simulation results were shown to demonstrate the proposed control strategies.

\section{References}




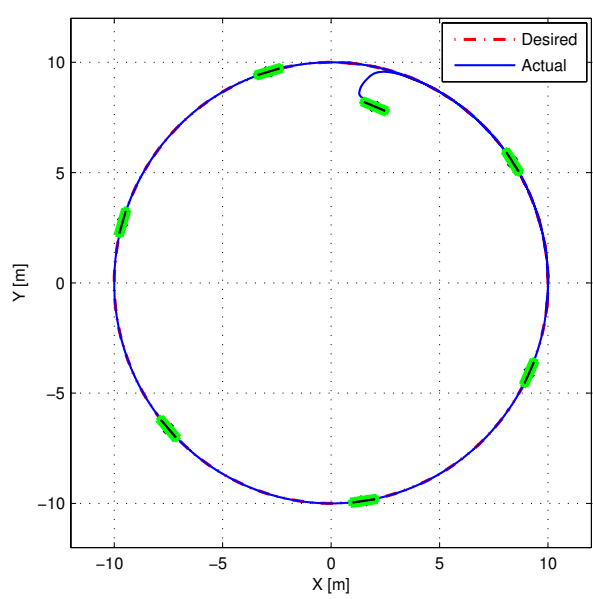

Fig. 6. Tracking demonstration in the XY-Plane (OFB)
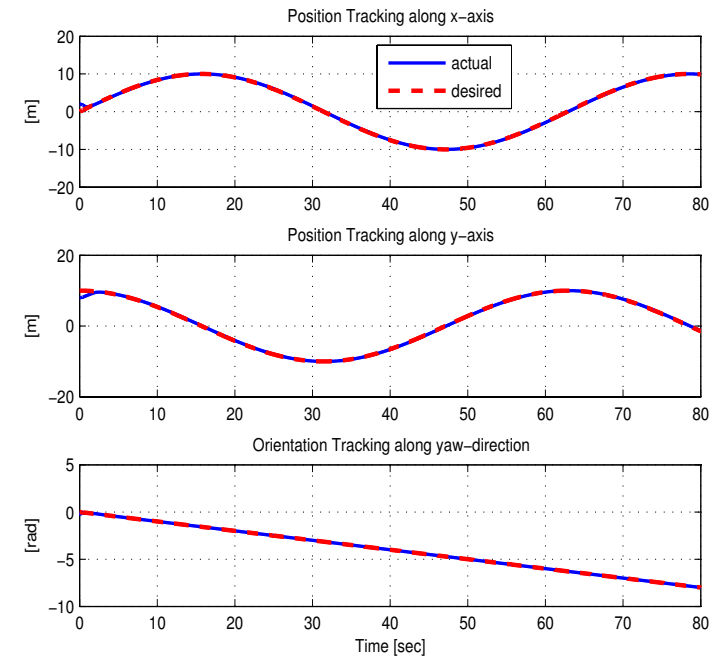

Fig. 7. Tracking demonstration (OFB)

[1] T. I. Fossen, Marine Control Systems : Guidance, Navigation, and Control of Ships, Rigs and Underwater Vehicles, Trondheim: Marine Cybernetics AS, Norway, 1st ed. 2002.

[2] R. Skjetne, O. N. Smogeli, and T. I. Fossen, "A Nonlinear Ship Manoeuvering Model: Identification and adaptive control with experiments for a model ship", Modeling, Identification and Control, Vol 25, No. 1, pp. 3-27, 2004.

[3] T. D. Nguyen, A. J. Sørensen, and S. T. Quek, "Design of Hybrid Controller for Dynamic Positioning from Calm to Extreme Sea Conditions", Automatica, Vol. 43, No. 5, pp. 768-785, May 2007.

[4] E. A. Tannuri and H.M. Morishita, "Experimental and numerical evaluation of a typical dynamic positioning system", Applied Ocean Research Vol. 28, No. 2, pp. 133-146, April 2006.

[5] F. Fahimi, "Nonlinear Model Predictive Formation Control for Groups of Autonomous Surface Vessels", International Journal of Control, Vol. 80, Issue 8, pp. 1248-1259, August 2007.

[6] K. D. Do and J. Pan, "Global tracking control of underactuated ships with nonzero off-diagonal terms in their system matrices", Automatica, Vol. 41, pp. 87 - 95, 2005.

[7] K. D. Do, "Global Robust and Adaptive Output Feedback
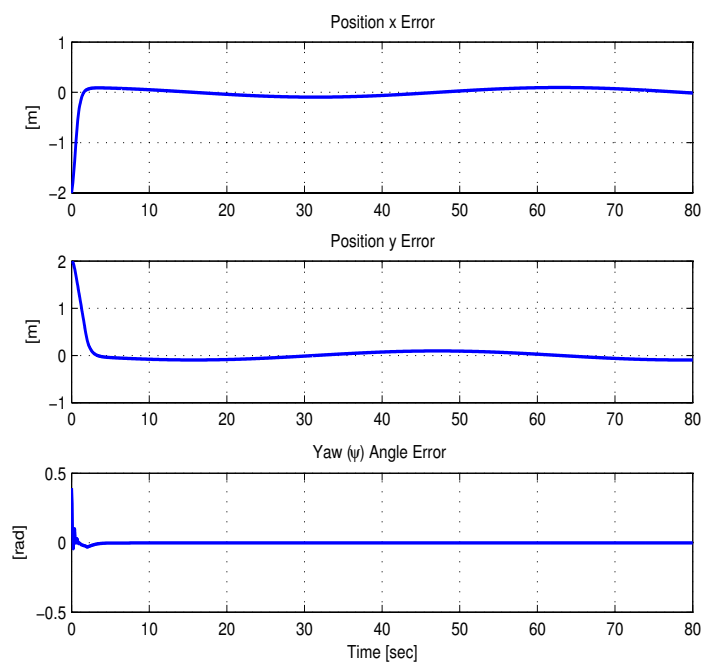

Fig. 8. Tracking Errors in Position and Yaw Angle (OFB)
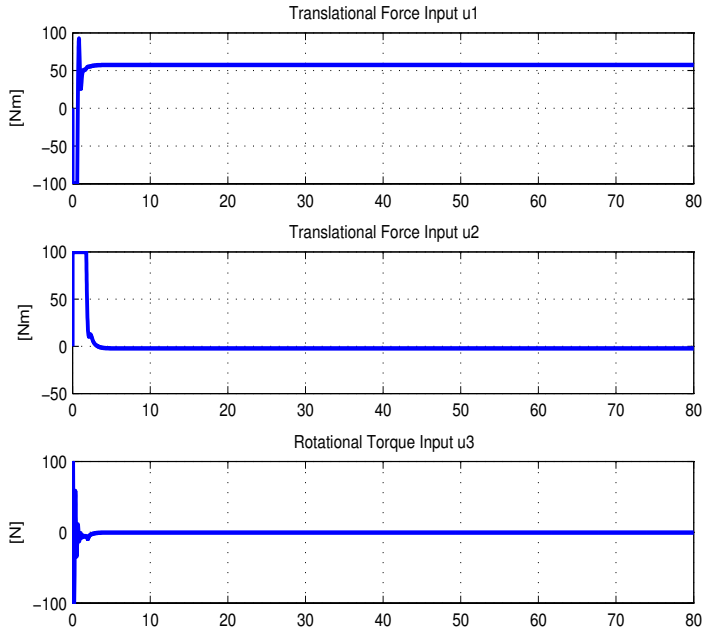

Fig. 9. Forces and Torque Input (OFB)

Dynamic Positioning of Surface Ships", IEEE International Conference on Robotics and Automation, Rome, Italy, pp. 4271 - 4276, April 2007.

[8] A. Behal, D. M. Dawson, W. E. Dixon, and Y. Fang, "Tracking and Regulation Control of an Underactuated Surface Vessel with Nonintegrable Dynamics", IEEE Transactions on Automatic Control, Vol. 47, No. 3, pp.495 - 500, March 2002.

[9] X. Zhang, A. Behal, D. M. Dawson, and B. Xian, "Output Feedback Control for a Class of Uncertain MIMO Nonlinear Systems with Non-Symmetric Input Gain Matrix", Proc. 44th IEEE Conference on Decision and Control, and the European Control Conference, Seville, Spain, pp. 7762 - 7767, Dec. 2005.

[10] J. Chen, A. Behal, D. M. Dawson, and X. Zhang, "Robust and Adaptive Output Feedback Control Strategies for a Class of Uncertain MIMO Nonlinear Systems", Technical Report, CRB, Clemson University, April 2006, Available: http://www.ece.clemson.edu/ece/crb/publictn/tr.htm.

[11] A. N. Atassi and H. K. Khalil, "A Separtion Principle for the Stabilization of a Class of Nonlinear Systems", IEEE Transactions on Automatic Control, Vol. 44, No. 9, pp.1672 1687, Sept. 1999. 